\title{
ON GROUPS OF CENTRAL TYPE, NON-DEGENERATE AND BIJECTIVE COHOMOLOGY CLASSES
}

\author{
NIR BEN DAVID AND YUVAL GINOSAR
}

\begin{abstract}
A finite group $G$ is of central type (in the non-classical sense) if it admits a non-degenerate cohomology class $[c] \in H^{2}\left(G, \mathbb{C}^{*}\right)(G$ acts trivially on $\left.\mathbb{C}^{*}\right)$. Groups of central type play a fundamental role in the classification of semisimple triangular complex Hopf algebras and can be determined by their representation-theoretical properties.

Suppose that a finite group $Q$ acts on an abelian group $A$ so that there exists a bijective 1 -cocycle $\pi \in Z^{1}(Q, \check{A})$, where $\check{A}=\operatorname{Hom}\left(\mathrm{A}, \mathbb{C}^{*}\right)$ is endowed with the diagonal $Q$-action. Under this assumption, Etingof and Gelaki gave an explicit formula for a non-degenerate 2-cocycle in $Z^{2}\left(G, \mathbb{C}^{*}\right)$, where $G:=A \rtimes Q$. Hence, the semidirect product $G$ is of central type.

In this paper we present a more general correspondence between bijective and non-degenerate cohomology classes. In particular, given a bijective class $[\pi] \in H^{1}(Q, \breve{A})$ as above, we construct non-degenerate classes $\left[c_{\pi}\right] \in H^{2}\left(G, \mathbb{C}^{*}\right)$ for certain extensions $1 \rightarrow A \rightarrow G \rightarrow Q \rightarrow 1$ which are not necessarily split. We thus strictly extend the above family of central type groups.
\end{abstract}

\section{INTRODUCTION}

It is well known that the dimension of an irreducible complex representation of a finite group $\Gamma$ cannot exceed $\sqrt{[\Gamma: Z(\Gamma)]}$, where $Z(\Gamma)$ is the center of $\Gamma$ (see e.g. [I, Corollary 2.30]). Classically, $\Gamma$ is termed "of central type" if it admits an irreducible representation $V$ of dimension $n:=\sqrt{[\Gamma: Z(\Gamma)]}$. However, here (following [AHN]) we call the quotient group $G:=\Gamma / Z(\Gamma)$ a group of central type.

Let $V$ be an $n$-dimensional irreducible representation of $\Gamma$. Since $Z(\Gamma)$ is mapped to the scalar matrices, the representation $V$ determines a projective representation $\bar{V}$ of $G$. Let $\left[c^{\prime}\right] \in H^{2}(G, Z(\Gamma))$ be the cohomology class corresponding to the extension $1 \rightarrow Z(\Gamma) \rightarrow \Gamma \rightarrow G \rightarrow 1$, and let $[c] \in H^{2}\left(G, \mathbb{C}^{*}\right)$ be the image of $\left[c^{\prime}\right]$ under the induced map $H^{2}(G, Z(\Gamma)) \rightarrow H^{2}\left(G, \mathbb{C}^{*}\right)$ (where $G$ acts trivially on $\mathbb{C}^{*}$ ). Then $[c]$ is associated to the projective representation $\bar{V}$ and the twisted group algebra $\mathbb{C}^{c}[G]$ is isomorphic to $M_{n}(\mathbb{C})$. It turns out [DMJ, Corollary 3] that the action of $G$ on $\mathbb{C}^{c}[G]$ by conjugation is isomorphic to the regular representation of $G$. This fact will be useful in the sequel. A 2-cocycle satisfying the above properties is called non-degenerate.

A remarkable result of Howlett and Isaacs asserts (assuming the classification of finite simple groups) that a group of central type $G$ is solvable [HI, Theorem 7.3].

Groups of central type have attracted considerable attention due to their representation-theoretical properties and their fundamental role in the theory of fusion symmetric categories via the theory of semisimple triangular Hopf algebras. More

Date: November 1, 2018.

Key words and phrases. Groups of central type, non-degenerate classes, bijective classes. 
specifically, it was proved that the isomorphism classes of semisimple triangular Hopf algebras of dimension $m$ over $\mathbb{C}$ are in bijection with the isomorphism classes of quadruples $(\Gamma, H, c, u)$ where $\Gamma$ is a group of order $m, H$ is a central type subgroup of $\Gamma, c$ is a non-degenerate 2-cocycle on $H$ and $u$ in an involution in $\Gamma$ [EG1,3]. The above bijection is given by twists for finite groups in the sense of Drinfeld [D]. Such a twist is supported on a subgroup of central type, and any finite dimensional semisimple triangular Hopf algebra over $\mathbb{C}$ is obtained from a group of central type equipped with a twist [EG3].

This motivates our interest in the explicit construction of central type groups. An important family of such groups was described in [EG2,3]: given a finite group $Q$ acting on an abelian group $A, \check{A}=\operatorname{Hom}\left(\mathrm{A}, \mathbb{C}^{*}\right)$ is a $Q$-module under the diagonal action (see (2.1)). Let $\pi: Q \rightarrow \check{A}$ be a bijective 1-cocycle, so that $\pi\left(g_{1} g_{2}\right)=\pi\left(g_{1}\right) g_{1}\left(\pi\left(g_{2}\right)\right)$ for every $g_{1}, g_{2} \in Q$, and $|Q|=|A|$. Etingof and Gelaki then construct a non-degenerate 2-cocycle $c$ on the semidirect product $G:=A \rtimes Q$, showing that $G$ is a group of central type. This non-degenerate 2-cocycle has an extra property: its restriction to $A$ is trivial.

Our main goal in this paper is to generalize the construction in [EG3] to extensions $1 \rightarrow A \rightarrow G \rightarrow Q \rightarrow 1$ which are not necessarily split. We also place the correspondence between bijective 1-cocycles $\pi: Q \rightarrow \breve{A}$ and non-degenerate 2 -cocycles on $G$ in a more general context, as follows.

Let

$$
[\beta]: 1 \rightarrow A \rightarrow G \rightarrow Q \rightarrow 1, \quad[\beta] \in H^{2}(Q, A)
$$

be an extension of a finite group $Q$ by a finite abelian group $A$. Let

$$
\operatorname{res}_{A}^{G}: H^{2}\left(G, \mathbb{C}^{*}\right) \rightarrow H^{2}\left(A, \mathbb{C}^{*}\right), \inf _{G}^{Q}: H^{2}\left(Q, \mathbb{C}^{*}\right) \rightarrow H^{2}\left(G, \mathbb{C}^{*}\right)
$$

be the restriction and inflation maps respectively, where $G$ acts trivially on $\mathbb{C}^{*}$.

As noted in the beginning of Section 4, if a 1-cocycle is bijective, so are all elements in its cohomology class; similarly for a non-degenerate 2-cocycle. Moreover, if $|A|=|Q|$ and $c: G \times G \rightarrow \mathbb{C}^{*}$ is a non-degenerate 2-cocycle, then so is $c \cdot f$ for every 2-cocycle $f$ inflated from $Q$. From now on we shall therefore refer also to cohomology classes (rather than cocycles) which are bijective or non-degenerate modulo the image of $\inf _{G}^{Q}$.

Our main result is:

Theorem A. Let (1.1) be an extension of finite groups such that $|A|=|Q|$. Then there is a 1-1 correspondence between bijective classes $[\pi] \in H^{1}(Q, \check{A})$ such that $[\beta] \cup[\pi]=0 \in H^{3}\left(Q, \mathbb{C}^{*}\right)$ and non-degenerate classes in $\operatorname{ker}\left(\operatorname{res}_{A}^{G}\right) \bmod \left[\operatorname{im}\left(\inf _{G}^{Q}\right)\right]$.

When $G=A \rtimes Q$, then clearly any $[\pi] \in H^{1}(Q, \check{A})$ satisfies $[\beta] \cup[\pi]=0$, and the correspondence in Theorem A yields the cohomology class of the non-degenerate 2 -cocycle that was constructed in [EG3].

Theorem A shows that if $A$ is a normal abelian subgroup of $G$ such that $|A|=$ $|G / A|$, then any non-degenerate class in $\operatorname{ker}\left(\operatorname{res}_{A}^{G}\right)$ is obtained, up to inflation, from a certain bijective class.

Further, fix any bijective class $[\pi] \in H^{1}(Q, \check{A})$. The correspondence in Theorem A gives rise to non-degenerate cohomology classes in $H^{2}\left(G, \mathbb{C}^{*}\right)$ for all extensions (1.1) such that $[\beta] \cup[\pi]=0$. We conclude: 
Corollary B. Let $A$ be a finite abelian group, $Q$ a finite group acting on $A$ and $[\pi] \in H^{1}(Q, \check{A})$ a bijective class. Then for every $[\beta] \in H^{2}(Q, A)$ such that $[\beta] \cup[\pi]=$ 0 , the group $G$ determined by the extension $[\beta]: 1 \rightarrow A \rightarrow G \rightarrow Q \rightarrow 1$ is of central type.

Corollary B gives a way to construct groups of central type from bijective cohomology classes. In practice, it yields groups of central type that cannot be constructed as a semidirect product in any way. In Section 5 we give an example of a non-split extension $[\beta]: 1 \rightarrow A \rightarrow G \rightarrow Q \rightarrow 1$ and a bijective class $[\pi] \in H^{1}(Q, \check{A})$ such that $[\beta] \cup[\pi]=0$. The group $G$ (of order 64 ) is hence of central type, but does not contain any abelian normal subgroup $N$ of order $|G / N|$ such that $G:=N \rtimes(G / N)$.

The 1-1 correspondence in Theorem A is obtained from an isomorphism of the quotient $\operatorname{ker}\left(\operatorname{res}_{A}^{G}\right) / \operatorname{im}\left(\inf _{G}^{Q}\right)$ and

$$
K_{\beta}:=\left\{[\pi] \in H^{1}(Q, \check{A}) \mid[\beta] \cup[\pi]=0 \in H^{3}\left(Q, \mathbb{C}^{*}\right)\right\} .
$$

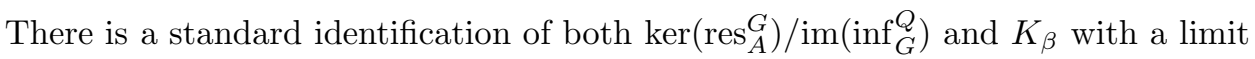
term of the Lyndon-Hochschild-Serre (LHS) spectral sequence (see 2.2 hereafter). We shall however give a self contained description of mutually inverse isomorphisms

$$
\left.\Pi: \operatorname{ker}\left(\operatorname{res}_{A}^{G}\right) / \operatorname{im}\left(\inf _{G}^{Q}\right) \rightarrow K_{\beta}, \quad C: K_{\beta} \rightarrow \operatorname{ker}\left(\operatorname{res}_{A}^{G}\right) / \operatorname{im}_{\left(\inf _{G}^{Q}\right)}\right)
$$

in terms of representatives and cocycles. This description will be convenient for establishing the correspondence in Theorem A.

The paper is organized as follows:

After some cohomological background in Section 2, we describe the mutually inverse isomorphisms $\Pi$ and $C$ in Section 3. These isomorphisms induce the 1-1 correspondence in Theorem A as shown in Section 4 The isomorphism $\Pi$, essentially that of $[\mathrm{K}$, Section 1.7], is interpreted here via the action of $Q$ on the primitive idempotents of the group algebra $\mathbb{C}[A]$. Section 5 is the example mentioned above. Finally, in Section [6 we apply the 1-1 correspondence between bijective and nondegenerate cohomology classes in Theorem A to construct twists for finite groups explicitly.

Acknowledgments. Part of this work is based on the M.Sc. thesis of the first author under the supervision of E. Aljadeff and S. Gelaki. We are grateful to them for their significant contribution. We also thank D. Blanc, H.W. Henn, M. Natapov, Á. del Río and U. Weiss for their help.

The first author was supported by Technion V.P.R. Fund - Dent Charitable Trust and by The Israel Science Foundation (grant No. 70/02-1).

\section{Preliminaries}

2.1. We first recall some elementary notation. Let $Q$ be a finite group acting on a finite abelian group $A$. This action induces a diagonal action of $Q$ on $\check{A}=$ $\operatorname{Hom}\left(\mathrm{A}, \mathbb{C}^{*}\right)$ defined by the pairing between $\check{A}$ and $A$ :

$$
\langle g(\chi), a\rangle=\left\langle\chi, g^{-1}(a)\right\rangle \in \mathbb{C}^{*}
$$

where $g \in Q, \chi \in \check{A}$ and $a \in A$ (the action on $\mathbb{C}^{*}$ is trivial).

Let (1.1) be an extension of $Q$ by $A$. Let $\{\bar{g}\}_{g \in Q}$ be a transversal set for $Q$ in $G$. Then any element in $G$ is uniquely expressed as $a \bar{g}$, where $a \in A$ and $g \in Q$. 
Recall that the multiplication in $G$ is determined by the conjugation of elements in $A$ via the given action

$$
\bar{g} a \bar{g}^{-1}=g(a), \quad a \in A, g \in Q,
$$

and by the 2-cocycle $\beta: Q \times Q \rightarrow A$ as follows

$$
\bar{g}_{1} \bar{g}_{2}=\beta\left(g_{1}, g_{2}\right) \overline{g_{1} g_{2}}, \quad g_{1}, g_{2} \in Q .
$$

We may further assume that $\beta$ is normalized, i.e.

$$
\beta(g, 1)=\beta(1, g)=1, \quad \forall g \in Q,
$$

by choosing the trivial element of $G$ as a representative of the trivial element in $Q$.

2.2. The second cohomology group $H^{2}(G, \check{A})$ admits the filtration

$$
\operatorname{im}\left(\inf _{G}^{Q}\right) \subseteq \operatorname{ker}\left(\operatorname{res}_{A}^{G}\right) \subseteq H^{2}(G, \check{A}),
$$

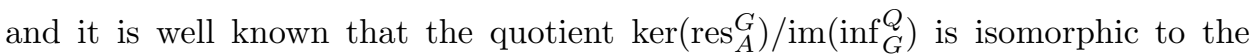
term $E_{\infty}^{1,1}$ in the LHS spectral sequence for the extension (1.1). Moreover, $E_{\infty}^{1,1}$ canonically embeds into $E_{2}^{1,1}$ as the kernel of the differential $d_{2}^{1,1}: E_{2}^{1,1} \rightarrow E_{2}^{3,0}$. Since $A$ is abelian and acts trivially on $\mathbb{C}^{*}$, we have

$$
E_{2}^{1,1}=H^{1}\left(Q, H^{1}\left(A, \mathbb{C}^{*}\right)\right)=H^{1}(Q, \check{A}), \quad E_{2}^{3,0}=H^{3}\left(Q, H^{0}\left(A, \mathbb{C}^{*}\right)\right)=H^{3}\left(Q, \mathbb{C}^{*}\right) .
$$

Furthermore, by [HS, Theorem 4], the differential $d_{2}^{1,1}$ in this case amounts to multiplication by the class of the extension up to sign. More precisely,

$$
\begin{aligned}
& d_{2}^{1,1}: H^{1}(Q, \check{A}) \rightarrow H^{3}\left(Q, \mathbb{C}^{*}\right) \\
& {[\pi] \mapsto-[\beta] \cup[\pi] .}
\end{aligned}
$$

where $[\beta] \in H^{2}(Q, A)$ is determined by the extension and $\cup$ is the usual cup product followed by the pairing $A \otimes \check{A} \rightarrow \mathbb{C}($ see (3.4) $)$. Both $K_{\beta}$ and $\operatorname{ker}\left(\operatorname{res}_{A}^{G}\right) / \operatorname{im}_{\left(\inf _{G}^{Q}\right)}$ may therefore be identified with $E_{\infty}^{1,1}$.

A detailed study of $d_{2}^{1,1}$, as well as of other low degree differentials in the general setup can be found in $[\mathrm{H}]$.

\section{The Isomorphisms $C$ And $\Pi$}

In this section we explicitly describe the mutually inverse isomorphisms $C$ : $K_{\beta} \rightarrow \operatorname{ker}\left(\operatorname{res}_{A}^{G}\right) / \operatorname{im}\left(\inf _{G}^{Q}\right)(\$ 3.1)$ and $\Pi: \operatorname{ker}\left(\operatorname{res}_{A}^{G}\right) / \operatorname{im}\left(\inf _{G}^{Q}\right) \rightarrow K_{\beta}(\$ 3.2)$.

3.1. We first construct the isomorphism $C: K_{\beta} \rightarrow \operatorname{ker}\left(\operatorname{res}_{A}^{G}\right) / \operatorname{im}\left(\inf _{G}^{Q}\right)$. Let $\pi \in$ $Z^{1}(Q, \check{A})$ be any 1-cocycle. Then it determines a 2-cochain $\varphi_{\pi} \in C^{2}\left(G, \mathbb{C}^{*}\right)$ as follows (compare with the construction for semidirect products [EG3, section 8]). For $\gamma_{1}=a_{1} \bar{g}_{1}, \gamma_{2}=a_{2} \bar{g}_{2} \in G$, let

$$
\varphi_{\pi}\left(\gamma_{1}, \gamma_{2}\right):=\left\langle\pi\left(g_{1}\right), g_{1}\left(a_{2}\right)\right\rangle^{-1} \in \mathbb{C}^{*} .
$$

Equivalently, by (2.1) and the 1-cocycle condition we obtain

$$
\varphi_{\pi}\left(\gamma_{1}, \gamma_{2}\right)=\left\langle\pi\left(g_{1}^{-1}\right), a_{2}\right\rangle .
$$

Note that for every $\pi_{1}, \pi_{2} \in Z^{1}(Q, \check{A})$

$$
\varphi_{\pi_{1} \pi_{2}}=\varphi_{\pi_{1}} \varphi_{\pi_{2}} .
$$

In general, the cochain $\varphi_{\pi}$ does not satisfy the cocycle condition. However, when $[\pi]$ is in $K_{\beta} \subseteq H^{1}(Q, \check{A})$, we can modify its construction to make it a cocycle, 
as follows. Recall that for any (left) $G$-module $M$, the $n$-th coboundary map $\delta_{G}^{n}: C^{n}(G, M) \rightarrow C^{n+1}(G, M)$ is defined by

$$
\begin{aligned}
\delta_{G}^{n}(\varphi)\left(\gamma_{1}, \ldots, \gamma_{n+1}\right):= & \gamma_{1} \cdot \varphi\left(\gamma_{2} \ldots, \gamma_{n+1}\right) \\
& +\sum_{i=1}^{n}(-1)^{i} \varphi\left(\gamma_{1}, \ldots, \gamma_{i} \gamma_{i+1}, \ldots, \gamma_{n+1}\right)+(-1)^{n+1} \varphi\left(\gamma_{1}, \ldots, \gamma_{n}\right),
\end{aligned}
$$

for every $\varphi$ in $C^{n}(G, M)$.

Proposition 3.1. For any $\pi \in Z^{1}(Q, \check{A})$ and any $a_{1} \bar{g}_{1}, a_{2} \bar{g}_{2}, a_{3} \bar{g}_{3} \in G$, $\delta_{G}^{2}\left(\varphi_{\pi}\right)\left(a_{1} \bar{g}_{1}, a_{2} \bar{g}_{2}, a_{3} \bar{g}_{3}\right)=\left\langle\pi\left(g_{1}\right), g_{1}\left(\beta\left(g_{2}, g_{3}\right)\right)\right\rangle^{-1}$.

Proof. This follows directly from the definition of $\delta_{G}^{2}$.

Next, let $[\pi] \in K_{\beta}$. Then $[\beta] \cup[\pi]=0 \in H^{3}\left(Q, \mathbb{C}^{*}\right)$ - i.e., the 3 -cochain

$$
\left(g_{1}, g_{2}, g_{3}\right) \mapsto\left\langle\pi\left(g_{1}\right), g_{1}\left(\beta\left(g_{2}, g_{3}\right)\right)\right\rangle
$$

is a 3 -coboundary on $Q[\mathrm{HS}, \mathrm{p} .118]$. Hence there exists a 2-cochain $\zeta_{\pi} \in C^{2}\left(Q, \mathbb{C}^{*}\right)$ such that

$$
\delta_{Q}^{2}\left(\zeta_{\pi}\right)\left(g_{1}, g_{2}, g_{3}\right)=\left\langle\pi\left(g_{1}\right), g_{1}\left(\beta\left(g_{2}, g_{3}\right)\right)\right\rangle, \forall g_{1}, g_{2}, g_{3} \in Q
$$

We now construct a 2-cocycle on $G$ from the cochain $\varphi_{\pi}$. Let

$$
c_{\pi}:=\varphi_{\pi} \bar{\zeta}_{\pi},
$$

where $\bar{\zeta}_{\pi} \in C^{2}\left(G, \mathbb{C}^{*}\right)$ is the inflation of $\zeta_{\pi}$ to $G$. By Proposition 3.1 and (3.5), we have $\delta_{G}^{2}\left(\varphi_{\pi} \bar{\zeta}_{\pi}\right)=0$. Consequently

$$
c_{\pi} \in Z^{2}\left(G, \mathbb{C}^{*}\right)
$$

We need the following

Proposition 3.2. Let $c_{\pi}$ be as above. Then:

(1) The restriction of $c_{\pi}$ to $A$ is cohomologically trivial.

(2) Any other choice of a 2-cochain $\zeta_{\pi}^{\prime} \in C^{2}\left(Q, \mathbb{C}^{*}\right)$ (satisfying (3.5)) yields a 2-cocycle $c_{\pi}^{\prime} \in Z^{2}\left(G, \mathbb{C}^{*}\right)$, with $c_{\pi}^{\prime} c_{\pi}^{-1}$ inflated from $Q$.

(3) Any other choice of a transversal set $\left\{\bar{g}^{\prime}\right\}_{g \in Q}$ for $Q$ in $G$ yields a 2-cocycle $c_{\pi}^{\prime} \in Z^{2}\left(G, \mathbb{C}^{*}\right)$, with $c_{\pi}^{\prime} c_{\pi}^{-1}$ inflated from $Q$.

(4) If $\pi \in B^{1}(Q, \check{A})$ then $c_{\pi} \in B^{2}\left(G, \mathbb{C}^{*}\right)$.

(5) For every $\pi_{1}, \pi_{2} \in Z^{1}(Q, \check{A})$, the 2-cocycle $c_{\pi_{1}} c_{\pi_{2}} c_{\pi_{1} \pi_{2}}^{-1}$ is inflated from $Q$.

Proof. (1) By (3.2), for every $a_{1}, a_{2} \in A$,

$$
\varphi_{\pi}\left(a_{1}, a_{2}\right)=\left\langle\pi(1), a_{2}\right\rangle=1 .
$$

Next, since the cochain $\bar{\zeta}_{\pi}$ is inflated from $Q$, its restriction to $A$ is a constant 2cocycle, and in particular a coboundary. It follows that the restriction of $c_{\pi}=\varphi_{\pi} \bar{\zeta}_{\pi}$ to $A$ is a coboundary.

(2) This holds since $\zeta_{\pi}^{\prime}$ differs from $\zeta_{\pi}$ by a 2-cocycle on $Q$.

(3) The two transversal sets differ by a 1-cochain $\lambda \in C^{1}(Q, A)$, i.e. $\bar{g}=\lambda(g) \bar{g}^{\prime}$ for every $g \in Q$. Let

$$
\gamma_{1}=a_{1} \overline{g_{1}}=a_{1} \lambda\left(g_{1}\right){\overline{g_{1}}}^{\prime}, \gamma_{2}=a_{2} \overline{g_{2}}=a_{2} \lambda\left(g_{2}\right){\overline{g_{2}}}^{\prime} \in G .
$$


Then

$$
\begin{aligned}
& c_{\pi}^{\prime}\left(\gamma_{1}, \gamma_{2}\right)=\left(\varphi_{\pi}^{\prime} \bar{\zeta}_{\pi}^{\prime}\right)\left(\gamma_{1}, \gamma_{2}\right)=\left\langle\pi\left(g_{1}^{-1}\right), a_{2} \lambda\left(g_{2}\right)\right\rangle \cdot \zeta_{\pi}^{\prime}\left(g_{1}, g_{2}\right)= \\
& =\left\langle\pi\left(g_{1}^{-1}\right), a_{2}\right\rangle \cdot\left\langle\pi\left(g_{1}^{-1}\right), \lambda\left(g_{2}\right)\right\rangle \cdot \zeta_{\pi}^{\prime}\left(g_{1}, g_{2}\right)= \\
& \left(\varphi_{\pi} \overline{\zeta_{\pi}}\right)\left(\gamma_{1}, \gamma_{2}\right) \cdot \zeta_{\pi}\left(g_{1}, g_{2}\right)^{-1} \cdot\left\langle\pi\left(g_{1}^{-1}\right), \lambda\left(g_{2}\right)\right\rangle \cdot \zeta_{\pi}^{\prime}\left(g_{1}, g_{2}\right)=c_{\pi}\left(\gamma_{1}, \gamma_{2}\right) \eta_{\pi}\left(\gamma_{1}, \gamma_{2}\right),
\end{aligned}
$$

where $\eta_{\pi} \in Z^{2}\left(G, \mathbb{C}^{*}\right)$ defined by

$$
\eta_{\pi}\left(\gamma_{1}, \gamma_{2}\right):=\zeta_{\pi}\left(g_{1}, g_{2}\right)^{-1} \cdot\left\langle\pi\left(g_{1}^{-1}\right), \lambda\left(g_{2}\right)\right\rangle \cdot \zeta_{\pi}^{\prime}\left(g_{1}, g_{2}\right)
$$

is inflated from $Q$.

(4) Suppose $\pi \in B^{1}(Q, \check{A})$. Then there is a $\chi_{\pi} \in \check{A}$ such that $\pi(g)=g\left(\chi_{\pi}\right) \chi_{\pi}^{-1}$ for every $g \in Q$. Setting $\zeta_{\pi}\left(g_{1}, g_{2}\right):=\left\langle\chi_{\pi}, \beta\left(g_{1}, g_{2}\right)\right\rangle$, we obtain

$$
\left\langle\pi\left(g_{1}\right), g_{1}\left(\beta\left(g_{2}, g_{3}\right)\right)\right\rangle=\left\langle g_{1}\left(\chi_{\pi}\right) \chi_{\pi}^{-1}, g_{1}\left(\beta\left(g_{2}, g_{3}\right)\right)\right\rangle=\delta_{Q}^{2}\left(\zeta_{\pi}\right)\left(g_{1}, g_{2}, g_{3}\right) .
$$

It follows that

$$
\begin{aligned}
c_{\pi}\left(a_{1} \bar{g}_{1}, a_{2} \bar{g}_{2}\right) & =\varphi_{\pi} \bar{\zeta}_{\pi}\left(a_{1} \bar{g}_{1}, a_{2} \bar{g}_{2}\right)=\left\langle\pi\left(g_{1}\right), g_{1}\left(a_{2}\right)\right\rangle^{-1}\left\langle\chi_{\pi}, \beta\left(g_{1}, g_{2}\right)\right\rangle \\
& =\left\langle g_{1}\left(\chi_{\pi}\right), g_{1}\left(a_{2}\right)\right\rangle^{-1}\left\langle\chi_{\pi}, g_{1}\left(a_{2}\right)\right\rangle\left\langle\chi_{\pi}, \beta\left(g_{1}, g_{2}\right)\right\rangle \\
& =\left\langle\chi_{\pi}, a_{1} a_{2}\right\rangle^{-1}\left\langle\chi_{\pi}, a_{1} g_{1}\left(a_{2}\right) \beta\left(g_{1}, g_{2}\right)\right\rangle=\delta_{G}^{1}\left(\bar{\chi}_{\pi}^{-1}\right)\left(a_{1} \bar{g}_{1}, a_{2} \bar{g}_{2}\right),
\end{aligned}
$$

where $\bar{\chi}_{\pi} \in C^{1}\left(G, \mathbb{C}^{*}\right)$ is defined by $\bar{\chi}_{\pi}(a \bar{g})=\chi_{\pi}(a)$. Hence, with the above choice of $\zeta_{\pi}$, we deduce that $c_{\pi} \in B^{2}\left(G, \mathbb{C}^{*}\right)$.

(5) This follows from the definition of $c_{\pi}$ (3.6) and from equation (3.3).

We summarize the above in:

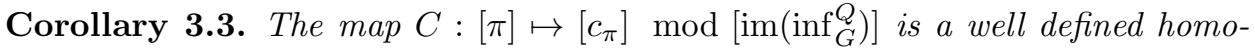
morphism from $K_{\beta}$ to $\operatorname{ker}\left(\operatorname{res}_{A}^{G}\right) / \operatorname{im}\left(\inf _{G}^{Q}\right)$.

3.2. We show that $C$ is actually an isomorphism by presenting its inverse map $\Pi: \operatorname{ker}\left(\operatorname{res}_{A}^{G}\right) / \operatorname{im}\left(\inf _{G}^{Q}\right) \rightarrow K_{\beta}$. A similar construction can be found in $[\mathrm{K}, \S 1.7]$.

Lemma 3.4. With the above notation, any class in $\operatorname{ker}\left(\operatorname{res}_{A}^{G}\right)$ admits a representative $c \in Z^{2}\left(G, \mathbb{C}^{*}\right)$ such that

$$
c\left(\gamma_{1}, \gamma_{2}\right)=c\left(a \gamma_{1}, \gamma_{2}\right)
$$

for all $\gamma_{1}, \gamma_{2} \in G$, and $a \in A$.

Proof. See [K, Lemma 7.1, P.59].

Let $c \in Z^{2}\left(G, \mathbb{C}^{*}\right)$ be a 2 -cocycle on $G$ such that $[c] \in \operatorname{ker}\left(\operatorname{res}_{A}^{G}\right)$. We need to construct a 1-cocycle on $Q$ with values in $\check{A}$. For this purpose we first show how $[c] \in \operatorname{ker}\left(\operatorname{res}_{A}^{G}\right)$ yields a character on $A$ for any $g \in Q$. By Lemma 3.4, we may assume $c\left(\gamma_{1}, \gamma_{2}\right)=c\left(a \gamma_{1}, \gamma_{2}\right)$ for all $\gamma_{1}, \gamma_{2} \in G$ and $a \in A$. Now, for any $a \in A$ and any $g \in Q$ define

$$
\begin{aligned}
\pi_{c}(g): A & \rightarrow \mathbb{C}^{*} \\
a & \mapsto c\left(\overline{g^{-1}}, a\right) .
\end{aligned}
$$

Proposition 3.5. Let $\pi_{c}(g)$ be as in (3.9). Then:

(1) For any $g \in Q, \pi_{c}(g) \in \check{A}$.

(2) $\pi_{c}(g)$ does not depend on the choice of the transversal set $\{\bar{g}\}_{g \in Q}$.

(3) The map $g \mapsto \pi_{c}(g)$ is a 1-cocycle from $Q$ to $\check{A}$.

(4) If $c^{\prime} \in[c]$ satisfies (3.8), then $\pi_{c^{\prime}} \pi_{c}^{-1}$ is a coboundary.

(5) If $c_{1}$ and $c_{2}$ satisfy (3.8), then so does $c_{1} c_{2}$. Moreover, $\pi_{c_{1} c_{2}}=\pi_{c_{1}} \pi_{c_{2}}$. 
(6) $\pi_{c} \in B^{1}(Q, \check{A})$ if and only if $[c] \in \operatorname{im}\left(\inf _{G}^{Q}\right)$.

(7) The 3-cochain $\left(g_{1}, g_{2}, g_{3}\right) \mapsto\left\langle\pi_{c}\left(g_{1}\right), g_{1}\left(\beta\left(g_{2}, g_{3}\right)\right)\right\rangle$ is a coboundary on $Q$.

Proof. See [K, Theorem 7.3, P.60] for the proof of (1)-(6). Let us prove (7). By Proposition [3.1. $\left\langle\pi\left(g_{1}\right), g_{1}\left(\beta\left(g_{2}, g_{3}\right)\right)\right\rangle=\delta_{G}^{2}\left(\varphi_{\pi}^{-1}\right)\left(a_{1} \bar{g}_{1}, a_{2} \bar{g}_{2}, a_{3} \bar{g}_{3}\right)$ for any $\pi \in$ $Z^{1}(Q, \check{A})$ and $a_{1} \bar{g}_{1}, a_{2} \bar{g}_{2}, a_{3} \bar{g}_{3} \in G$. We claim that in case $\pi=\pi_{c}$, we can substitute the $G$-cochain $\varphi_{\pi_{c}}$ with an appropriate $Q$-cochain. Indeed,

$$
\left.\varphi_{\pi_{c}}\left(a_{1} \bar{g}_{1}, a_{2} \bar{g}_{2}\right)=\left\langle\pi_{c}\left(g_{1}\right), g_{1}\left(a_{2}\right)\right\rangle^{-1}=\left\langle\pi_{c}\left(g_{1}^{-1}\right), a_{2}\right)\right\rangle=c\left(\overline{g_{1}}, a_{2}\right) .
$$

Applying the 2-cocycle condition, we obtain

$$
\begin{aligned}
& c\left(\overline{g_{1}}, a_{2}\right)=c\left(\overline{g_{1}}, a_{2} \overline{g_{2}}\right) c\left(\overline{g_{1}} a_{2}, \overline{g_{2}}\right)^{-1} c\left(a_{2}, \overline{g_{2}}\right)= \\
& =c\left(\overline{g_{1}}, a_{2} \overline{g_{2}}\right) c\left(\bar{g}_{1}\left(a_{2}\right) \overline{g_{1}}, \overline{g_{2}}\right)^{-1} c\left(a_{2}, \overline{g_{2}}\right) .
\end{aligned}
$$

Since $c$ satisfies (3.8), then equations (3.10) and (3.11) imply

$$
\varphi_{\pi_{c}}\left(a_{1} \bar{g}_{1}, a_{2} \bar{g}_{2}\right)=c\left(a_{1} \overline{g_{1}}, a_{2} \overline{g_{2}}\right) c\left(\overline{g_{1}}, \overline{g_{2}}\right)^{-1} .
$$

Note that since $c$ is a 2-cocycle on $G$, we have $\delta_{G}^{2}(c)=0$. From (3.12) we obtain that

$$
\delta_{G}^{2}\left(\varphi_{\pi_{c}}\right)\left(a_{1} \bar{g}_{1}, a_{2} \bar{g}_{2}, a_{3} \bar{g}_{3}\right)=\delta_{Q}^{2}\left(\tilde{c}^{-1}\right)\left(g_{1}, g_{2}, g_{3}\right),
$$

where $\tilde{c} \in C^{2}\left(Q, \mathbb{C}^{*}\right)$ is defined by $\tilde{c}\left(g, g^{\prime}\right):=c\left(\bar{g}, \bar{g}^{\prime}\right), \quad g, g^{\prime} \in Q$. In particular,

$$
\left\langle\pi_{c}\left(g_{1}\right), g_{1}\left(\beta\left(g_{2}, g_{3}\right)\right)\right\rangle=\delta_{Q}^{2}(\tilde{c})\left(g_{1}, g_{2}, g_{3}\right) .
$$

We summarize the above.

Corollary 3.6. The map $\Pi:[c] \bmod \left[\operatorname{im}\left(\inf _{G}^{Q}\right)\right] \mapsto\left[\pi_{c}\right]$ is a well defined homomorphism from $\operatorname{ker}\left(\operatorname{res}_{A}^{G}\right) / \operatorname{im}_{\left(\inf _{G}^{Q}\right)}^{Q}$ to $K_{\beta} \subseteq H^{1}(Q, \check{A})$.

To complete the discussion we have:

Proposition 3.7. The homomorphisms $C$ and $\Pi$ in Corollaries 3.3 and 3.6 are mutually inverse.

Proof. Let $[\pi] \in K_{\beta}$. Note that since $\beta$ is normalized, then by (3.5) we have

$$
\delta_{Q}^{2}\left(\zeta_{\pi}\right)(g, 1, g)=1
$$

for every $g \in Q$. Developing $\delta_{Q}^{2}$ we obtain that

$$
\zeta_{\pi}(1, g)=\zeta_{\pi}(g, 1), \forall g \in Q .
$$

We first claim that the choice of the cochain $\zeta_{\pi}$ satisfying (3.5) can be done such that

$$
\zeta_{\pi}(1, g)=\zeta_{\pi}(g, 1)=1, \forall g \in Q
$$

Indeed, let

$$
\zeta_{\pi}^{\prime}\left(g_{1}, g_{2}\right):=\zeta_{\pi}\left(g_{1}, g_{2}\right) \zeta_{\pi}\left(1, g_{2}\right)^{-1}
$$

Then clearly

$$
\zeta_{\pi}^{\prime}(1, g)=1
$$

for every $g \in Q$. To complete the proof of the claim, we need to check that $\zeta_{\pi}^{\prime}$ satisfies equation (3.5). Indeed, for every $g_{1}, g_{2}, g_{3} \in Q$,

$$
\begin{aligned}
& \delta_{Q}^{2}\left(\zeta_{\pi}^{\prime}\right)\left(g_{1}, g_{2}, g_{3}\right)=\delta_{Q}^{2}\left(\zeta_{\pi}\right)\left(g_{1}, g_{2}, g_{3}\right) \zeta_{\pi}^{-1}\left(1, g_{2} g_{3}\right) \zeta_{\pi}\left(1, g_{2}\right)= \\
& \delta_{Q}^{2}\left(\zeta_{\pi}\right)\left(g_{1}, g_{2}, g_{3}\right) \delta_{Q}^{2}\left(\zeta_{\pi}^{-1}\right)\left(1, g_{2}, g_{3}\right)=\left\langle\pi\left(g_{1}\right), g_{1}\left(\beta\left(g_{2}, g_{3}\right)\right)\right\rangle\left\langle\pi(1), \beta\left(g_{2}, g_{3}\right)\right\rangle^{-1}= \\
& =\left\langle\pi\left(g_{1}\right), g_{1}\left(\beta\left(g_{2}, g_{3}\right)\right)\right\rangle\left(=\delta_{Q}^{2}\left(\zeta_{\pi}\right)\left(g_{1}, g_{2}, g_{3}\right)\right) .
\end{aligned}
$$


Note that the 2-cocycle $c_{\pi}:=\varphi_{\pi} \bar{\zeta}_{\pi}$ satisfies condition (3.8), and hence $\pi_{c_{\pi}}$ is well defined. By the definitions (3.2), (3.6) and (3.9), we have that for every $g \in Q$ and $a \in A$,

$$
\left\langle\pi_{c_{\pi}}(g), a\right\rangle=c_{\pi}\left(\overline{g^{-1}}, a\right)=\langle\pi(g), a\rangle \cdot \zeta_{\pi}\left(g^{-1}, 1\right) .
$$

Choosing $\zeta_{\pi}$ that satisfies (3.14), we obtain $\left\langle\pi_{c_{\pi}}(g), a\right\rangle=\langle\pi(g), a\rangle$. By Proposition 3.2(2) and Proposition 3.5) (6) we deduce that

$$
\left[\pi_{c_{\pi}}\right]=[\pi] .
$$

Conversely, let $c \in Z^{2}\left(G, \mathbb{C}^{*}\right)$ be as in Lemma 3.4. Then for every $g_{1}, g_{2} \in Q$ and $a_{1}, a_{2} \in A$,

$$
c_{\pi_{c}}\left(a_{1} \bar{g}_{1}, a_{2} \bar{g}_{2}\right)=\varphi_{\pi_{c}}\left(a_{1} \bar{g}_{1}, a_{2} \bar{g}_{2}\right) \zeta_{\pi_{c}}\left(g_{1}, g_{2}\right) .
$$

By equation (3.12),

$$
c_{\pi_{c}}\left(a_{1} \bar{g}_{1}, a_{2} \bar{g}_{2}\right)=c\left(a_{1} \overline{g_{1}}, a_{2} \overline{g_{2}}\right) c\left(\overline{g_{1}}, \overline{g_{2}}\right)^{-1} \zeta_{\pi_{c}}\left(g_{1}, g_{2}\right) .
$$

This shows that $c$ and $c_{\pi_{c}}$ differ by a 2-cocycle which is inflated from $Q$. Thus,

$$
\left[c_{\pi_{c}}\right]=[c] \bmod \left[\operatorname{im}_{\left.\left(\inf _{G}^{Q}\right)\right] .}\right.
$$

3.3. We can interpret the 1-cocycle $\pi_{c}$ as interchanging the primitive idempotents of $\mathbb{C}[A]$ by $Q$ via the conjugation in $\mathbb{C}^{c}[G]$ as in Proposition 3.8 below. This will be needed in the next section. First, some notation:

Let $c \in Z^{2}\left(G, \mathbb{C}^{*}\right)$ be any 2-cocycle and let $\mathbb{C}^{c}[G]$ be the corresponding twisted group algebra with a $\mathbb{C}$-basis $\left\{U_{\gamma}\right\}_{\gamma \in G}$ (satisfying $\left.U_{\sigma} U_{\gamma}=c(\sigma, \gamma) U_{\sigma \gamma}\right)$. Then $\gamma \in G$ acts on $\mathbb{C}^{c}[G]$ by left conjugation with $U_{\gamma}$ :

$$
\gamma \cdot U_{\sigma}=U_{\gamma} U_{\sigma} U_{\gamma}^{-1}=[\sigma, \gamma]_{c} U_{\gamma \sigma \gamma^{-1}}
$$

where

$$
[\sigma, \gamma]_{c}:=c(\gamma, \sigma) c\left(\gamma \sigma \gamma^{-1}, \gamma\right)^{-1}=c\left(\gamma, \sigma \gamma^{-1}\right) c\left(\sigma \gamma^{-1}, \gamma\right)^{-1} .
$$

Next, let $[\beta]: 1 \rightarrow A \rightarrow G \rightarrow Q \rightarrow 1$ be an extension of finite groups, and let $c \in Z^{2}\left(G, \mathbb{C}^{*}\right)$ be any 2 -cocycle. Then $G$ acts on the central primitive idempotents of the subalgebra $\mathbb{C}^{\operatorname{res}_{A}^{G}(c)}[A] \subseteq \mathbb{C}^{c}[G]$ by conjugation. For every $\gamma \in G$ and a central primitive idempotent $\iota \in \mathbb{C}^{\mathrm{res}_{A}^{G}(c)}[A]$,

$$
\gamma(\iota)=U_{\gamma} \cdot \iota \cdot U_{\gamma}^{-1}
$$

Suppose now that $A$ is abelian and $[c] \in \operatorname{ker}\left(\operatorname{res}_{A}^{G}\right)$. By Lemma 3.4 we may assume that $c$ satisfies (3.8). In this case the commutative subalgebra $\mathbb{C}[A]=$ $\mathbb{C}^{\mathrm{res}_{A}^{G}(c)}[A] \subseteq \mathbb{C}^{c}[G]$ admits the primitive idempotents

$$
\iota_{\chi}=\frac{1}{|A|} \sum_{a \in A}\langle\chi, a\rangle^{-1} U_{a}
$$

for every $\chi \in \check{A}$. The action of $G$ on the set of primitive idempotents is via the quotient $Q$. Then the 1-cocycle $\pi_{c}$ associated to $c$ determines this action as follows.

Proposition 3.8. With the above notation let $g \in Q$ and $\chi \in \check{A}$. Then

$$
g\left(\iota_{\chi}\right)=\iota_{g(\chi) \pi_{c}(g)} .
$$


Proof.

$$
\begin{aligned}
g\left(\iota_{\chi}\right) & =U_{\bar{g}} \iota_{\chi} U_{\bar{g}}^{-1}=\frac{1}{|A|} \sum_{a \in A}\langle\chi, a\rangle^{-1} U_{\bar{g}} U_{a} U_{\bar{g}}^{-1} \\
& =\frac{1}{|A|} \sum_{a \in A}\langle\chi, a\rangle^{-1}[a, \bar{g}]_{c} U_{g(a)}=\frac{1}{|A|} \sum_{a \in A}\langle\chi, a\rangle^{-1}\left\langle\pi_{c}\left(g^{-1}\right), a\right\rangle \cdot U_{g(a)} \\
& =\frac{1}{|A|} \sum_{a \in A}\left\langle g(\chi) \pi_{c}(g), g(a)\right\rangle^{-1} U_{g(a)}=\iota_{g(\chi) \pi_{c}(g)} .
\end{aligned}
$$

Remark 3.9. This construction of the map $\Pi$ in 33.2 works also if $\mathbb{C}^{*}$ is replaced by any $A$-trivial $G$-module. In particular, assume $G$ as above acts on a field $K$, with $A$ acting trivially, and let $c \in Z^{2}\left(G, K^{*}\right)$ be a 2-cocycle such that $[c] \in \operatorname{ker}\left(\operatorname{res}_{A}^{G}\right)$. One can then establish (3.17) for the action of $Q$ on the primitive idempotents of $K[A]$ in the crossed product $K^{c} *[G]$.

\section{Bijective and Non-Degenerate Cohomology Classes}

In this section we prove:

(1) If $\pi: Q \rightarrow \check{A}$ is a bijective 1-cocycle such that $[\pi] \in K_{\beta}$, then $c_{\pi} \in$ $Z^{2}\left(G, \mathbb{C}^{*}\right)$ is a non-degenerate 2-cocycle (Theorem 4.4).

(2) If $c \in Z^{2}\left(G, \mathbb{C}^{*}\right)$ is a non-degenerate 2-cocycle satisfying (3.8) (in particular $[c] \in \operatorname{ker}\left(\operatorname{res}_{A}^{G}\right)$ ) and if $|A|=|Q|$, then $\pi_{c}$ is bijective (Theorem 4.6).

This will establish the correspondence in Theorem A between bijective classes

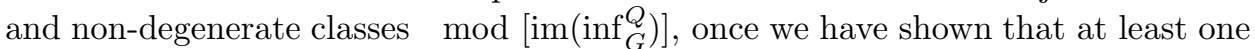
of the two properties is independent of the choice of cocycle. We therefore prove:

Proposition 4.1. Let $\pi \in Z^{1}(Q, M)$, where $M$ is any $Q$-module. If $\pi$ is surjective, then so are all the cocycles in its cohomology class.

Proof. Let $\pi^{\prime} \in Z^{1}(Q, M)$ such that $\left[\pi^{\prime}\right]=[\pi]$. Then there exists $m \in M$ such that for every $g \in Q, \pi^{\prime}(g)=\pi(g) g(m) m^{-1}$. By the hypothesis, $m=\pi\left(g^{\prime}\right)$ for some $g^{\prime} \in Q$. Then

$$
\pi^{\prime}(g)=\pi(g) g\left(\pi\left(g^{\prime}\right)\right) m^{-1}=\pi\left(g g^{\prime}\right) m^{-1} .
$$

Hence $\pi^{\prime}$ is surjective as well.

Let $[\beta]: 1 \rightarrow A \rightarrow G \rightarrow Q \rightarrow 1$ be an extension of finite groups, where $A$ is abelian. We first prove that if $\pi: Q \rightarrow \check{A}$ is a bijective 1-cocycle (in particular $|Q|=|A|)$ such that $[\pi] \in K_{\beta}$, then the twisted group algebra $\mathbb{C}^{c_{\pi}}[G]$ is isomorphic as a $G$-module by conjugation (3.15) to the regular left representation $\mathbb{C}[G]$ of $G$. As mentioned in the introduction, this will show that $c_{\pi} \in Z^{2}\left(G, \mathbb{C}^{*}\right)$ is a nondegenerate 2-cocycle [DMJ, Corollary 3].

Let $c \in Z^{2}\left(G, \mathbb{C}^{*}\right)$ be any 2-cocycle and let $\left\{U_{\gamma}\right\}_{\gamma \in G}$ be a $\mathbb{C}$-basis of the left $G$-module $\mathbb{C}^{c}[G]$, where $\gamma \in G$ acts by conjugation (3.15). Further, let $\{\gamma\}_{\gamma \in G}$ be a $\mathbb{C}$-basis of the left $G$-module $\mathbb{C}[G]$, where $\gamma \in G$ acts by left multiplication. We present two morphisms between these $G$-modules, which are mutually inverse in case $c=c_{\pi}$ for a bijective 1-cocycle $\pi$ such that $[\pi] \in K_{\beta}$ and hence yield the $G$-module isomorphism between $\mathbb{C}^{c_{\pi}}[G]$ and $\mathbb{C}[G]$. 
With the notation of (3.16) we define:

$$
\begin{aligned}
\psi_{c}: \mathbb{C}^{c}[G] & \rightarrow \mathbb{C}[G] \\
U_{\gamma} & \mapsto \sum_{\sigma \in G}\left[\gamma, \sigma^{-1}\right]_{c} \sigma
\end{aligned}
$$

and

$$
\begin{aligned}
\theta_{c}: \mathbb{C}[G] & \rightarrow \mathbb{C}^{c}[G] \\
\sigma & \mapsto \frac{1}{|G|} \sum_{\gamma \in G}\left[\gamma, \sigma^{-1}\right]_{c}^{-1} U_{\gamma}
\end{aligned}
$$

(and extend $\psi_{c}$ and $\theta_{c}$ linearly).

Proposition 4.2. The maps $\psi_{c}$ and $\theta_{c}$ are $G$-module morphisms.

Proof. For any $\gamma, \tau \in G$ we have

$$
\begin{aligned}
& \psi_{c}\left(\tau \cdot U_{\gamma}\right)=[\gamma, \tau]_{c} \psi_{c}\left(U_{\tau \gamma \tau^{-1}}\right)=[\gamma, \tau]_{c} \sum_{\sigma \in \Gamma}\left[\tau \gamma \tau^{-1}, \sigma^{-1}\right]_{c} \sigma= \\
& \sum_{\sigma \in \Gamma}\left[\gamma, \sigma^{-1} \tau\right]_{c} \sigma=\sum_{\eta \in \Gamma}\left[\gamma, \eta^{-1}\right]_{c} \tau \eta=\tau \sum_{\eta \in \Gamma}\left[\gamma, \eta^{-1}\right]_{c} \eta=\tau \psi_{c}\left(U_{\gamma}\right) .
\end{aligned}
$$

Hence $\psi_{c}$ is a $G$-module map.

Next,

$$
\theta_{c}(\tau)=\frac{1}{|G|} \sum_{\gamma \in G}\left[\gamma, \tau^{-1}\right]_{c}^{-1} U_{\gamma}=\frac{1}{|G|} \sum_{\gamma \in G}\left[\tau \gamma \tau^{-1}, \tau^{-1}\right]_{c}^{-1} U_{\tau \gamma \tau^{-1}}=
$$

by definition (3.16)

$$
\begin{aligned}
& =\frac{1}{|G|} \sum_{\gamma \in G} c\left(\tau^{-1}, \tau \gamma \tau^{-1}\right)^{-1} c\left(\gamma, \tau^{-1}\right) U_{\tau \gamma \tau^{-1}}= \\
& \frac{1}{|G|} \sum_{\gamma \in G} c\left(1, \gamma \tau^{-1}\right)^{-1} c\left(\tau, \gamma \tau^{-1}\right) c\left(\tau^{-1}, \tau\right)^{-1} c\left(\gamma, \tau^{-1}\right) U_{\tau \gamma \tau^{-1}}= \\
& \frac{1}{|G|} \sum_{\gamma \in G} c\left(\tau, \gamma \tau^{-1}\right) c\left(\tau^{-1}, \tau\right)^{-1} c\left(\gamma, \tau^{-1}\right) c(\gamma, 1)^{-1} U_{\tau \gamma \tau^{-1}}= \\
& \frac{1}{|G|} \sum_{\gamma \in G} c\left(\tau, \gamma \tau^{-1}\right) c\left(\gamma \tau^{-1}, \tau\right)^{-1} U_{\tau \gamma \tau^{-1}}=
\end{aligned}
$$

again, by $\underline{3.16}$

$$
=\frac{1}{|G|} \sum_{\gamma \in G}[\gamma, \tau]_{c} U_{\tau \gamma \tau^{-1}}=\frac{1}{|G|} \sum_{\gamma \in G} \tau \cdot U_{\gamma}=\tau \cdot \theta_{c}(1) .
$$

Thus,

$$
\theta_{c}(\sigma \tau)=\sigma \tau \cdot \theta_{c}(1)=\sigma \cdot\left(\tau \cdot \theta_{c}(1)\right)=\sigma \cdot \theta_{c}(\tau)
$$

and $\theta_{c}$ is a $G$-module map. This completes the proof of the proposition.

We now give a sufficient condition on the 2-cocycle $c$ for $\psi_{c}$ and $\theta_{c}$ to be inverse to each other.

Proposition 4.3. Let $c \in Z^{2}\left(G, \mathbb{C}^{*}\right)$ be such that for any non-trivial $\tau \in G$, $\sum_{\gamma \in G}[\gamma, \tau]_{c}=0$. Then $\psi_{c}$ is invertible with $\psi_{c}^{-1}=\theta_{c}$. In particular, $c$ is nondegenerate.

Proof. Since $\operatorname{dim}_{\mathbb{C}} \mathbb{C}^{c}[G]=\operatorname{dim}_{\mathbb{C}} \mathbb{C}[G]=|G|$, it is enough to show that $\theta_{c}$ is a right inverse of $\psi_{c}$. We first compute $\psi_{c} \circ \theta_{c}$ on the trivial element $1 \in G$.

$$
\left(\psi_{c} \circ \theta_{c}\right)(1)=\psi_{c}\left(\frac{1}{|G|} \sum_{\gamma \in G} U_{\gamma}\right)=\frac{1}{|G|} \sum_{\gamma \in G} \sum_{\sigma \in G}\left[\gamma, \sigma^{-1}\right]_{c} \sigma .
$$


By the hypothesis, $\frac{1}{|G|} \sum_{\gamma \in G}\left[\gamma, \sigma^{-1}\right]_{c}=\delta_{1, \sigma}$. Thus

$$
\left(\psi_{c} \circ \theta_{c}\right)(1)=\sum_{\sigma \in G} \delta_{1, \sigma} \sigma=1 .
$$

Consequently,

$$
\left(\psi_{c} \circ \theta_{c}\right)(\tau)=\psi_{c}\left(\theta_{c}(\tau)\right)=\psi_{c}\left(\tau \cdot \theta_{c}(1)\right)=\tau \psi_{c}\left(\theta_{c}(1)\right)=\tau \cdot 1=\tau .
$$

Theorem 4.4. Let $\pi: Q \rightarrow \check{A}$ be a bijective 1-cocycle such that $[\pi] \in K_{\beta}$, then $c_{\pi} \in Z^{2}\left(G, \mathbb{C}^{*}\right)$ is a non-degenerate 2-cocycle.

Proof. We show that $c_{\pi} \in Z^{2}(G, \check{A})$ satisfies the criterion in Proposition 4.3 Recall that for every $a_{1} \bar{g}_{1}, a_{2} \bar{g}_{2} \in G$,

$$
c_{\pi}\left(a_{1} \bar{g}_{1}, a_{2} \bar{g}_{2}\right)=\left\langle\pi\left(g_{1}^{-1}\right), a_{2}\right\rangle \zeta_{\pi}\left(g_{1}, g_{2}\right),
$$

where $\zeta_{\pi} \in C^{2}\left(Q, \mathbb{C}^{*}\right)$ satisfies (3.5). Let $a \bar{g}$ be a non-trivial element in $\mathrm{G}$. We prove that $\sum_{\gamma \in G}[\gamma, a \bar{g}]_{c_{\pi}}=0$.

$$
\begin{gathered}
\sum_{a^{\prime} \bar{g}^{\prime} \in G}\left[a^{\prime} \bar{g}^{\prime}, a \bar{g}\right]_{c_{\pi}}=\sum_{a^{\prime} \bar{g}^{\prime} \in G} c_{\pi}\left(a \bar{g}, a^{\prime} \bar{g}^{\prime}\right) c_{\pi}\left((a \bar{g}) a^{\prime} \bar{g}^{\prime}(a \bar{g})^{-1}, a \bar{g}\right)^{-1}= \\
\sum_{a^{\prime} g^{\prime} \in G}\left\langle\pi\left(g^{-1}\right), a^{\prime}\right\rangle \zeta_{\pi}\left(g, g^{\prime}\right)\left\langle\pi\left(g g^{\prime-1} g^{-1}\right), a\right\rangle^{-1} \zeta_{\pi}\left(g g^{\prime} g^{-1}, g\right)^{-1}= \\
\left(\sum_{a^{\prime} \in A}\left\langle\pi\left(g^{-1}\right), a^{\prime}\right\rangle\right) \cdot\left(\sum_{g^{\prime} \in Q} \zeta_{\pi}\left(g, g^{\prime}\right)\left\langle\pi\left(g g^{\prime-1} g^{-1}\right), a\right\rangle^{-1} \zeta_{\pi}\left(g g^{\prime} g^{-1}, g\right)^{-1}\right) .
\end{gathered}
$$

If $g$ is not trivial in $Q$ then the injectivity of $\pi$ implies that $\pi\left(g^{-1}\right)$ is not the trivial character in $\check{A}$. Consequently

$$
\sum_{a^{\prime} \in A}\left\langle\pi\left(g^{-1}\right), a^{\prime}\right\rangle=0, \quad g \neq 1 .
$$

Suppose then that $g=1$. Applying (3.13) we obtain

$$
\begin{aligned}
& \sum_{g^{\prime} \in Q} \zeta_{\pi}\left(g, g^{\prime}\right)\left\langle\pi\left(g g^{-1} g^{-1}\right), a\right\rangle^{-1} \zeta_{\pi}\left(g g^{\prime} g^{-1}, g\right)^{-1}= \\
& \sum_{g^{\prime} \in Q}\left\langle\pi\left(g^{\prime-1}\right), a\right\rangle^{-1} .
\end{aligned}
$$

Since $\pi: Q \rightarrow \check{A}$ is bijective, we have

$$
\sum_{g^{\prime} \in Q}\left\langle\pi\left(g^{\prime-1}\right), a\right\rangle^{-1}=\sum_{\chi \in \check{A}}\langle\chi, a\rangle^{-1}=|A| \delta_{a, 1} .
$$

From (4.4) and (4.6) we see that $\sum_{\gamma \in G}[\gamma, a \bar{g}]_{c_{\pi}}$ vanishes unless both $g$ and $a$ are trivial.

We now prove the other direction of the correspondence in Theorem A.

Let $[\beta]: 1 \rightarrow A \rightarrow G \rightarrow Q \rightarrow 1$ be an extension of finite groups, and let $c \in Z^{2}\left(G, \mathbb{C}^{*}\right)$ be any 2 -cocycle. Let $\iota$ be any central primitive idempotent of the subalgebra $\mathbb{C}^{\operatorname{res}_{A}^{G}(c)}[A] \subseteq \mathbb{C}^{c}[G]$. It is easily checked that $\mathbb{C}^{c}[G]\left(\bigoplus_{\gamma \in G} \gamma(\iota)\right)$ is a two sided ideal of $\mathbb{C}^{c}[G]$. We obtain a necessary condition for the simplicity of $\mathbb{C}^{c}[G]$, or equivalently, for the non-degeneracy of $c$. See [AS, Lemma 1.2] for a more general setup. 
Proposition 4.5. If a 2-cocycle $c \in Z^{2}\left(G, \mathbb{C}^{*}\right)$ is non-degenerate, then $G$ acts transitively on the set of central primitive idempotents of the subalgebra $\mathbb{C}^{\mathrm{res}}{ }_{A}^{G}(c)[A]$ for any normal subgroup $A \triangleleft G$.

Assume again that $A$ is abelian and $[c] \in \operatorname{ker}\left(\operatorname{res}_{A}^{G}\right)$ satisfies (3.8). Substituting $\iota_{1}=\frac{1}{|A|} \sum_{a \in A} U_{a}$, the primitive idempotent of $\mathbb{C}[A]$ which corresponds to the trivial character $1_{A} \in \check{A}$ into equation (3.17) we obtain

$$
g\left(\iota_{1}\right)=\iota_{\pi_{c}(g)} .
$$

Finally, equation (4.7) and Proposition 4.5 imply:

Theorem 4.6. Let $1 \rightarrow A \rightarrow G \rightarrow Q \rightarrow 1$ be an extension of finite groups with $A$ abelian and let $c \in Z^{2}\left(G, \mathbb{C}^{*}\right)$ be a non-degenerate 2-cocycle satisfying (3.8). Then $\pi_{c} \in Z^{1}(Q, \check{A})$ is surjective. In particular, if $|A|=|Q|$, then $\pi_{c}$ is bijective.

This completes the proof of Theorem A.

\section{EXAMPLE}

The following example was shown to us by E. Aljadeff and Á. del Río. Let $Q:=C_{4} \times C_{2}=\langle\sigma\rangle \times\langle\tau\rangle$ act on $A:=C_{4} \times C_{2}=\langle x\rangle \times\langle y\rangle$ as follows:

$$
\sigma(x)=x y, \quad \tau(x)=x^{-1}, \quad \sigma(y)=\tau(y)=y .
$$

Let $\check{A}:=\langle\check{x}\rangle \times\langle\check{y}\rangle$, where $\check{x}: \begin{array}{lll}x & \mapsto & i \\ y & \mapsto & 1\end{array}$ and $\check{y}: \begin{array}{rrr}x & \mapsto & 1 \\ y & \mapsto & -1\end{array}$. Define:

$$
\begin{aligned}
\pi: Q & \rightarrow \check{A} \\
\sigma^{k} \tau^{l} & \mapsto \check{x}^{l-(-1)^{l} k} \check{y}^{l} .
\end{aligned}
$$

One can easily check that $\pi$ is a bijective 1-cocycle.

Next, consider the extension:

$$
[\beta]: 1 \rightarrow A \rightarrow G:=\langle x, y, \bar{\sigma}, \bar{\tau}\rangle \rightarrow Q \rightarrow 1
$$

determined by

$$
\bar{\sigma}^{4}=\bar{\tau}^{2}=1, \quad \bar{\tau} \bar{\sigma} \bar{\tau}^{-1} \bar{\sigma}^{-1}=x^{-1} y .
$$

Then $[\beta] \cup[\pi]=0$, and by Corollary B, the group $G$ is of central type. However, there is no abelian normal subgroup $N \triangleleft G$ of order 8 such that $G:=N \rtimes(G / N)$. Corollary B therefore allows us to construct central type groups which could not be obtained by [EG3].

\section{Twists for Finite Groups}

Theorem A has a useful application to the explicit construction of twists for finite groups. Let $\phi: \mathbb{C}[G] \rightarrow C^{\prime}$ be a $G$-module isomorphism between the regular $G$-module $\mathbb{C}[G]$ and a $G$-coalgebra $\left(C^{\prime}, \triangle^{\prime}, \varepsilon^{\prime}\right)$. A result of Movshev [M, Proposition $5]$ says that $C^{\prime}$ is isomorphic as a $G$-coalgebra to the coalgebra $\mathbb{C}[G]_{J_{\phi}}$, where

$$
J_{\phi}=\left(\phi^{-1} \otimes \phi^{-1}\right) \triangle^{\prime}(\phi(1))
$$

is a twist for $G$.

Let $[\beta]: 1 \rightarrow A \rightarrow G \rightarrow Q \rightarrow 1$ be an extension, and suppose that $[\pi] \in H^{1}(Q, \check{A})$ is a bijective class such that $[\beta] \cup[\pi]=0$. 
Let $c_{\pi}$ be a 2 -cocycle on $G$ determined by $\pi$ as in equation (3.6) above. We apply (6.1) to the $G$-coalgebra $C^{\prime}=\left(\mathbb{C}^{c_{\pi}}[G]\right)^{*}$ by a $G$-module isomorphism $\phi_{\pi}$ : $\mathbb{C}[G] \rightarrow\left(\mathbb{C}^{c_{\pi}}[G]\right)^{*}$ described as follows. Equation (4.2) above yields an explicit $G$-module homomorphism $\theta_{c_{\pi}}$ between the regular module $\mathbb{C}[G]$ and $\mathbb{C}^{c_{\pi}}[G]$ with the conjugation action. Under the above assumption on $\pi$, the proof of Theorem 4.4 (claiming that $c_{\pi}$ is non-degenerate) shows that $\theta_{c_{\pi}}$ is actually an isomorphism. The element $r_{\pi}=\theta_{c_{\pi}}(1) \in \mathbb{C}^{c_{\pi}}[G]$ is regular, that is $\left\{\gamma\left(r_{\pi}\right)\right\}_{\gamma \in G}$ is a $\mathbb{C}$-basis of $\mathbb{C}^{c_{\pi}}[G]$. Similarly, the element $r_{\pi}^{*} \in\left(\mathbb{C}^{c_{\pi}}[G]\right)^{*}$ defined by:

$$
r_{\pi}^{*}\left(\gamma\left(r_{\pi}\right)\right)=\delta_{\gamma, e}
$$

generates a $\mathbb{C}$-basis of $\left(\mathbb{C}^{c_{\pi}}[G]\right)^{*}$. Then

$$
\begin{aligned}
\phi_{\pi}: \mathbb{C}[G] & \rightarrow\left(\mathbb{C}^{c_{\pi}}[G]\right)^{*} \\
\gamma & \mapsto \gamma\left(r_{\pi}^{*}\right)
\end{aligned}
$$

is a $G$-module isomorphism and $J_{\phi_{\pi}}$ is a twist for $G$.

\section{REFERENCES}

[AHN] E. Aljadeff, D. Haile and M. Natapov, Projective bases of division algebras and groups of central type, Israel Journal of Mathematics 146 (2005), 317-335.

[AS] E. Aljadeff and J. Sonn, Projective Schur algebras have abelian splitting fields, Journal of Algebra 175 (1995), 179-187.

[D] V.G. Drinfeld, Almost cocommutative Hopf algebras, Leningrad Mathematical Journal 1 (1990), no. 2, 321-342.

[DMJ] F.R. DeMeyer and G.J. Janusz, Finite groups with an irreducible representation of large degree, Mathematische Zeitschrift 108 (1969), 145-153.

[EG1] P. Etingof and S. Gelaki, Some properties of finite-dimensional semisimple Hopf algebras, Mathematical Research Letters 5 (1998), 191-197.

[EG2] P. Etingof and S. Gelaki, A method of construction of finite-dimensional triangular semisimple Hopf algebras, Mathematical Research Letters 5 (1998), 551-561.

[EG3] P. Etingof and S. Gelaki, The classification of triangular semisimple and cosemisimple Hopf algebras over an algebraically closed field, International Mathematics Research Notices $\mathbf{5}$ (2000), 223-234.

[H] J. Huebschmann, Automorphisms of group extensions and differentials in the LyndonHochschild-Serre spectral sequence, Journal of Algebra 72 (1981), no. 2, 296-334.

[HI] R. B. Howlett and I. M. Isaacs, On groups of central type, Mathematische Zeitschrift 179 (1982), 555-569.

[HS] G. Hochschild and J.-P. Serre, Cohomology of group extensions. Transactions of the American Mathematical Society 74, (1953). 110-134.

[I] I. M. Isaacs, Character theory of finite groups, Academic Press, New York-London, 1976.

$[\mathrm{K}] \quad$ G. Karpilovsky, Group representations. Vol. 2., North-Holland Publishing Co., Amsterdam, 1993.

[M] M. Movshev, Twisting in group algebras of finite groups, Functional Analysis and its Applications 27 (1994), 240-244.

Department of Mathematics, Technion-Israel Institute of Technology, Haifa 32000 , ISRAEL

E-mail address: benda@tx.technion.ac.il

Department of Mathematics, University of Haifa, Haifa 31905, Israel

E-mail address: ginosar@math.haifa.ac.il 